\title{
Development of androgenic polyembrioids in the anther culture of alloplasmic introgression lines of (H. vulgare) - T. aestivum and (H. marinum ssp. gussoneanum) $-T$. aestivum
}

\author{
L.A. Pershina ${ }^{1 *}$, N.V. Trubacheeva ${ }^{1}$, T.S. Osadchaya ${ }^{1}$, I.A. Belan ${ }^{2}$, L.P. Rosseeva ${ }^{2}$ \\ 1 Institute of Cytology and Genetics, SB RAS, Novosibirsk, Russia \\ ${ }^{2}$ Omsk Agricultural Scientific Center, Omsk, Russia
}

DOI 10.18699/ICG-PlantGen2019-66

(c) Autors, 2019

* e-mail: pershina@bionet.nsc.ru

\begin{abstract}
The peculiarities of the development of embryo-like structures and regenerants in anther culture of introgression alloplasmic lines of common wheat carrying the cytoplasm of barley were compared with euplasmic lines. In all genotypes, the predominant formation of polyembryoids was found, out of which seedling families developed. According to a study of phenotypic traits and the level of fertility in androgenic regenerants with a spontaneously doubled number of chromosomes and restored fertility, about $80 \%$ of families contained clones. Sister DH lines formed from clones and included in breeding are simultaneously tested in different ecological zones.

Key words: alloplasmic lines; androgenesis; doubled haploid; clones.
\end{abstract}

\section{Introduction}

Introgressive hybridization is the basic method for increasing the genetic diversity of cultivated plants (Gill et al., 2011). An additional source of biodiversity can also be alloplasmic lines (allo-lines) with restored fertility (Liu et al., 2016), which are formed as a result of the replacement of the cytoplasm with an alien one. New intergenomic interactions in these lines may cause epigenetic modification of nuclear genes (Soltani et al., 2016) and changes in the manifestation of signs (Liu et al., 2002). We have developed an approach to obtain introgressive doubled haploid (DH) allo-lines of bread wheat which are used in genetic studies (Trubacheeva et al., 2012) and in breeding (Pershina et al., 2018). It was shown that one of the limitations in obtaining the genetic diversity of DH lines of triticale via anther culture is the development of a large number of clones among androgenic regenerants (Oleszczuk et al., 2014). How often clones are formed as a result of the cultivation of anthers of other genotypes is not known, since the works where this problem has been studied are few. In this report, the results of studying this phenomenon in anther culture of introgression allo-lines of bread wheat with the cytoplasm of $H$. vulgare and $H$. marinum ssp. gussoneanum $(2 n=28)$ are presented.

\section{Materials and methods}

The formation of single seedlings and their families was studied in anther culture of nine introgression allo-lines with the cytoplasm of $H$. vulgare and three introgression euplasmic lines of bread wheat. All these lines have different combinations of genes for resistance to fungal pathogens, introgressed into wheat from its wild relatives. In addition, two allo-lines with the cytoplasm of $\mathrm{H}$. marinum and chromosome $7 \mathrm{H}^{\text {mar }}$ were used. Anther culture was performed according to previously used methods (Osadchaya et al., 2017). The frequency of individual embryo-like structures which are sources for regeneration of individual seedlings and their families was analyzed.

\section{Results and discussion}

In the anther culture of all studied lines, embryo-like structures were formed from microspores (Figure 1,a). Separate embryo-like structures are formed into polyembryos. The family of seedlings developed from one polyembryoid (see Figure $1, b$ ). The family of albinos and the family of green seedlings are presented in Figure 1, $c$ and $d$. The formation of androgenic polyembryos was described for certain varieties of bread wheat (Seldimirova et al., 2016). In our work, it has been shown that in the studied introgressive allo-lines and euplasmic lines polyembryoids are formed predominantly.

In total, in all the lines 208 androgenic embryo-like structures have shown the ability to regenerate green seedlings. Of these, $174(83.6 \%)$ embryo-like structures resulted in seedling families. In allo-lines with the cytoplasm of $H$. vulgare the frequency of embryo-like structures, from which the family of green seedlings developed, was $81.8 \%$ with the variation of this indicator in different lines from 63.6 to $100 \%$. In allo-lines with the cytoplasm of $H$. marinum $90.4 \%$ of the embryo-like structures gave the families of regenerants. In euplasmic lines, the value of this indicator is $87.5 \%$.

Thus, regardless of the origin of alloplasmic and euplasmic introgressive lines via anther culture polyembryoids mainly formed, of which families involving from two to 22 seedlings were developed. Among the 34 single androgenic plants that reached heading, $24(70.5 \%)$ were completely sterile, three plants set single seeds and seven plants were fertile with a seed set between 15 and 35 per ear. Phenotypic traits and fertility levels were studied in plants of 121 families of allo-lines. Within 99 families, the plants were phenotypically identical and did not differ from each other in fertility. Plants within these families can be assigned to clones. Most families, 68 out of $90(75.5 \%)$, had either completely sterile plants (53 families), or besides completely sterile plants, included plants with single grains (from 1 to 3) (15 families). Plants from these families are of lower growth than the original plants, anther donors, and they can be assigned to haploids. 

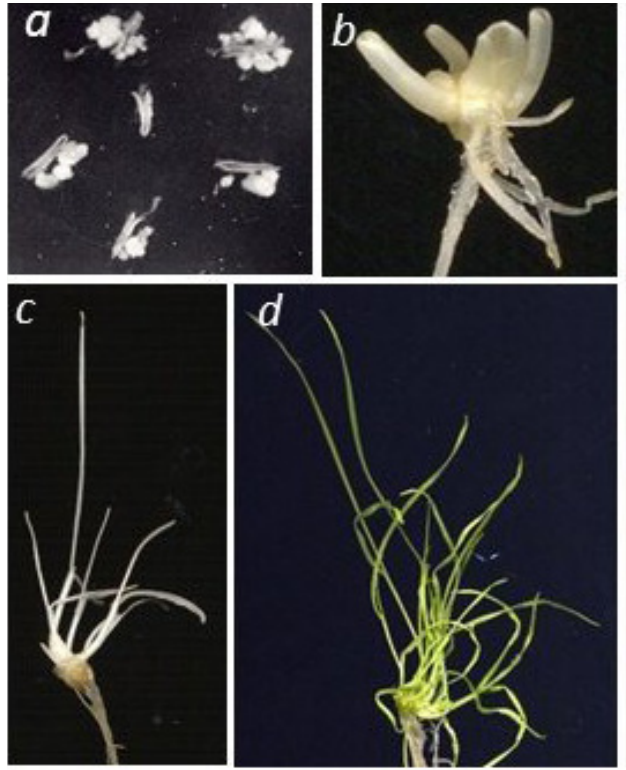
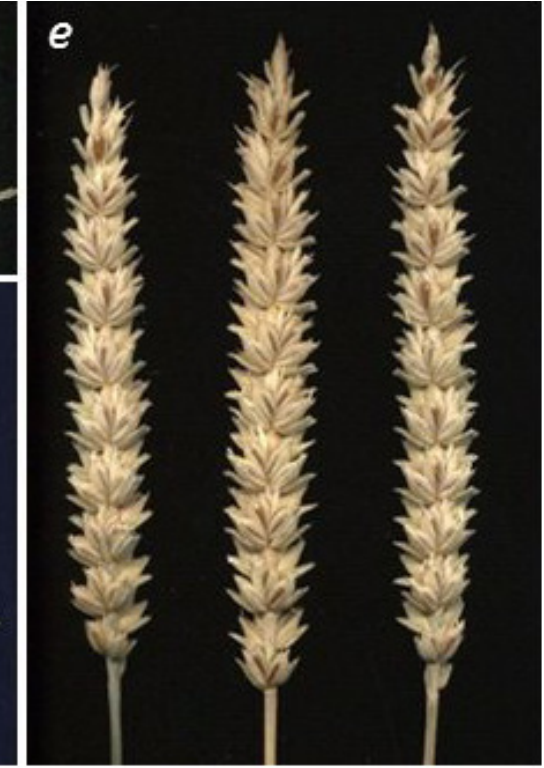

Figure 1. The embryo-like structures in anther culture $(a)$; the regeneration of the seedlings from a polyembryoids (b); the family of albino $(c)$ and green $(d)$ seedlings; ears of sister regenerants from the same family with restored fertility $(e)$.
Of these, 53 families ( $53.5 \%$ ) had only sterile plants and 15 families, in addition to completely sterile plants, included separate plants with single grains (from 1 to 3 ). Sterile plants in these families are identical in phenotype, and they can be assigned to haploids compared to taller and fertile donor plants. Due to the spontaneous doubling of the number of chromosomes via androgenesis, plants of 31 families were fertile. In four families, the plants set from 5 to 10 grains per ear; in five families, from 10 to 20 grains per ear. Plants in 22 families contained from 20 to more than 30 grains per ear (see Figure 1,e).

The remaining 22 families out of 121 studied included plants that differed in fertility and phenotypic traits. These families included completely sterile plants, plants with low and high levels of fertility. Based on a comparison of fertility and phenotypic traits, it can be assumed that in 99 families out of 121 studied clones were formed, the frequency being $81.8 \%$. From the seeds of individual fertile plants and plants from families, DH lines are formed, which are included in cytogenetic and molecular analysis for a more detailed study of the putative clones and families with plants of different fertility.

\section{Conclusions}

Our results showed that, regardless of the origin of the cytoplasm, introgression lines of common wheat in the anther culture of individual microspores form not individual androgenic embryoids, but polyembryoids from which plant families (clusters) develop. According to phenotypic characteristics and fertility levels, families of regenerants, which are considered as clones, have been previously isolated. A more detailed analysis of these families will determine how effective the selection of clones by phenotype and level of fertility is. The sister DH lines included in breeding are simultaneously tested for resistance to fungal pathogens and agronomically valuable traits in different ecological zones. The results of such tests will determine the expediency of the practical use of clones to obtain DH lines.

\section{References}

Gill B.S., Friebe B.R., Frank F. White F.F. Alien introgressions represent a rich source of genes for crop improvement. PNAS. 2011;19: 7657-7658. DOI 10.1073/pnas.1104845108.

Liu C.G., Wu Y.W., Hou H., Zhang C., Zhang Y., McIntosh R.A. Value and utilization of alloplasmic common wheats with Aegilops crassa cytoplasm. Plant Breed. 2002;121(5):407-410. DOI 10.1046/j. 1439-0523.2002.755374.x.

Liu Y., Tang L., Xu Q., Ma D., Zhao M., Sun J., Chen W. Experimental and genomic evidence for the indica-type cytoplasmic effect in Oryza sativa L. ssp. japonica. J Integrative Agriculture. 2016;15(10): 2183-2191. DOI 10.1016/S2095-3119(15)61190-X.

Oleszczuk S., Tyrka M., Zimny J. The origin of clones among androgenic regenerants of hexaploid triticale. Euphytica. 2014:198(3): 325-336. DOI 10.1007/s10681-014-1109-1.

Osadchaya T.S., Trubacheeva N.V., Kravtsova L.A., Belan I.A., Rosseeva L.P., Pershina L.A. Study of fertility and cytogenetic variability in androgenic plants $\left(\mathrm{R}_{0}\right.$ and $\left.\mathrm{R}_{1}\right)$ of the alloplasmic introgression lines of common wheat. Russian J Genetics: Applied Research. 2017:7(3):318-326. DOI 10.1134/S2079059717030121.

Pershina L.A., Belova L.I., Trubacheeva N.V., Osadchaya T.S., Shumny V.K., Belan I.A., Rosseeva L.P., Nemchenko V.V., Abakumov S.N. Alloplasmic recombinant lines (H. vulgare)-T. aestivum with 1RS.1BL translocation: initial genotypes for production of common wheat varieties. Vavilov J Gen Breed. 2018;22(5):544-552. DOI 10.18699/VJ18.393.

Seldimirova O.A., Titova G.T., Kruglova N.N. A complex morphohistological approach to the in vitro study of morphogenic structures in a wheat anther culture. Biol Bull. 2016:43(2):121-126. DOI $10.1134 /$ S1062359016020084.

Soltani A., Kumar A., Mergoum M., Pirseyedi S.M., Hegstad J.B., Mazaheri M., Kianian S.F. Novel nuclear-cytoplasmic interaction in wheat (Triticum aestivum) induces vigorous plants. Funct Integr Gen. 2016;16(2):171-182. DOI 10.1007/s10142-016-0475-2.

Trubacheeva N.V., Kravtsova L.A., Devyatkina E.P., Efremova T.T., Sinyavskaya M.G., Shumny V.K., Pershina L.A. Heteroplasmic and homoplasmic states of mitochondrial and chloroplast DNA regions in progenies of distant common wheat hybrids of different origins. Russ. J. Genet.: Appl. Res. 2012:2(6):494-500.

Acknowledgements. The work is supported by project No. 0324-20190039, RFBR grant No. 17-04-01738.

Conflict of interest. The authors declare no conflict of interest. 